\title{
Ethical Standards in Sport and Exercise Science Research: 2014 Update
}

D. J. Harriss, G. Atkinson

Bibliography

DOI http://dx.doi.org/

$10.1055 / \mathrm{s}-0033-1358756$

Int J Sports Med 2013; 34:

1025-1028 @ Georg Thieme

Verlag KG Stuttgart · New York

ISSN 0172-4622

\section{Correspondence}

\section{J. Harriss}

Research Institute for Sport and Exercise Sciences

Henry Cotton Campus

Liverpool John Moores

University

Webster Street

Liverpool L3 2ET

United Kingdom

Tel.: + 44/15/19 046236

Fax: +44/15/12 073224

D.Harriss@ljmu.ac.uk
For publication in the International Journal of Sports Medicine (IJSM), studies involving human participants or animals must have been conducted in accordance with recognised ethical standards and national/international laws. At the very first submission stage, authors are required to confirm that these standards and laws have been adhered to by reading, and formally citing, this editorial within the methods section of their own manuscript. Authors who do not provide any information regarding ethical approval will have their manuscripts rejected before it enters the peer-review process, without any option to resubmit.

In the original 2009 IJSM editorial [3], we described the ethical considerations embedded into national/international laws and provided specific guidance on the ethical issues which commonly arise in Sports Medicine research. In 2011, this information was updated to recognise the ethical principles of other professional associations and treaties when conducting research involving human participants [4]. Additional information was also provided on the use of Laboratory Animals in research, and on the links between sample size and research ethics. In our new update for 2014 onwards, we elaborate on,

A. The ethical issues relating to the investigation of doping agents

B. The use of animals for answering research questions that appear to be solely focussed on the enhancement of athletic performance

C. Sample size and the issue of the burden to individual research participants.

\section{Research Using Human Participants}

Authors who cite this editorial confirm that research using human participants has been conducted ethically according to the principles of the Declaration of Helsinki [2]. The Declaration is intended to be read as a whole and every principle is equally important, but those points mostcommonly considered by sport and exercise scientists are summarised below.

1. Basic principles. Respect the rights and welfare of participants which must take precedence over all other interests.

2. Ethical review. Before research begins and before amendments are applied, research must be reviewed and approved by an appropriate ethics committee.

3. The research protocol. The study, research design and statistical analysis must be clearly described, justifiable and appropriate. In draw- ing up the research protocol, the researcher must;

a) consider ethical issues in accordance with the Declaration of Helsinki,

b) provide information regarding funding, sponsors, institutional affiliations, other potential conflicts of interest,

c) consider the contribution to new knowledge and consider the environment,

d) include details of any incentives for participants and provisions for treating and/or compensating participants who are harmed as a consequence of participation in the research study,

e) describe the arrangements for post-study access by all participants to interventions identified as beneficial in the study or access to other appropriate care or benefits.

4. Consent. Informed consent/assent should be provided freely by the participant and should ideally be in writing. If written consent/assent cannot be obtained, or is not appropriate, then oral consent/assent should be formally documented and witnessed. Research that involves children or other populations that cannot consent (e.g. vulnerable populations) should seek consent from an appropriate person and assent from the participant. Research involving participants who are physically or mentally incapable of giving consent may be undertaken only if the physical or mental condition that prevents giving informed consent is a necessary characteristic of the research population. Informed consent/assent must include the

a) aims of the research,

b) methods,

c) sources of funding,

d) conflicts of interest,

e) institutional affiliations,

f) anticipated benefits and potential risks,

g) potential discomfort and

h) right to refuse to participate or withdraw consent without reprisal.

5. Conduct. Research must be conducted

a) in accordance with appropriate risk management,

b) by appropriately qualified researchers and support staff,

c) with skill and care,

d) in an appropriate setting,

e) in order to protect the privacy of participants and confidentiality of their personal information and

f) in accordance with laws and regulations of the country or countries in which the 
research is to be performed as well as international norms and standards. Specific laws relevant to research ethical may regulate the collection, use and/or storage of human tissue; the protection of individuals that lack the capacity to consent; data protection; and the use of drugs in research.

6. Governance. Serious adverse events occurring during the study must be reported to the ethics committee that ethically reviewed and approved the research.

Authors may conduct their research in accordance with principles detailed by professional associations and treaties other than the World Medical Association Declaration of Helsinki such as the International Sociological Association's (ISA) Code of Ethics [11]. This update recognises that differences in ethical principles may exist between professional associations. For example, the ISA's code of Ethics states that "The consent of research subjects and informants should be obtained in advance. Covert research should be avoided in principle, unless it is the only method by which information can be gathered, and/or when access to the usual sources of information is obstructed by those in power." [11]. Suggesting that consent in sociological research is less stringent than in experimental research [7]. Authors are required to confirm whether aspects of their research abides by ethical principles proclaimed by professional associations or treaties that differ in status to the Declaration of Helsinki.

By reading and citing this editorial the author confirms the following [7]:

1. Consent to participation was valid, such that the participants were provided with adequate information, the consent was given voluntarily and that those providing consent were competent to do so.

2. If research was carried out on participants who were vulnerable or not competent to give consent then the authors confirm that the participants were appropriately identified, there was justification for carrying out the research on these individuals and additional measures were put in place to ensure the research was ethical.

3. Issues of privacy and confidentiality have been considered beyond what is legally required. Where "privacy is the protection of control over information about oneself; control over access to oneself, both physically and mentally; and control over ones ability to make important decisions about family and lifestyle in order to be self expressive and to develop varied relationships" [7]. And confidentiality is when the "participant discloses to the researcher information which the participant regards as confidential or secret [default assumption]; and the researcher undertakes (implicitly or explicitly) not to reveal this information to anyone who does not already possess it." [7].

4. Researchers have considered their legal and ethical obligations to breach privacy and confidentiality.

5. If information provided confidentially as part of a research study has been used for other purposes that confidentiality has been preserved by anonymising the information or by seeking the participant's consent.

6. Risks relating to harm, inconvenience, time and money and benefits to the participant, to other individuals, to the researchers and organisations have been considered, balanced, communicated to the participants and appropriately managed when relevant.

7. Participants have not been exploited and particular groups discriminated from participating in the research.
8. There are appropriate governance arrangements and structures in place if participants are asked to donate biological material for use in future research, such as a biobank. This should involve appropriate consideration of broad consent, privacy and confidentiality, feedback to the participant of incidental findings, storage of material, commercial involvement, donor involvement and intellectual property rights.

\section{Research Involving Animals}

$\nabla$

Authors who cite this editorial confirm that research involving animals has been conducted ethically according to the principles of the Guide for the Care and Use of Laboratory Animals of the Institute for Laboratory Animal Research [8]. Again, the guide is intended to be read as a whole, but the basic obligations on the researcher are summarised below. The researcher must;

1. Ensure the appropriateness of experimental methods

2. Legally acquire animals

3. Ensure that animals are properly housed and fed to ensure safe, hygienic and comfortable living conditions.

4. Maintain a record of animal care

5. Ensure that animal maintenance and research are carried out by qualified personnel, following all legal statutes and regulations

6. Administer appropriate pain management to minimize suffering, discomfort and pain

The eighth edition of the Guide for the Care and Use of Laboratory Animals [9], published in 2010, includes expanded coverage of the ethics of laboratory animal use; components of effective Animal Care and Use Programs; and new guidelines for the housing, environment, and enrichment of terrestrial and aquatic animals, and for veterinary and clinical care [10]. Specifically:

1. The core foundation of the guide - replacement, refinement, and reduction.

2. An Animal Care and Use Program

3. The performance standards approach for animal care and care practices

4. The care and use of fish and other aquatic species

5. Housing space and enclosures for animals' social needs

6. Environmental enrichment got the enhancement of animal well-being to provide sensory and motor stimulation and promote psychological health

7. Discussion of animal biosecurity practices

\section{Update. Exercise protocols in animal research $\nabla$}

A useful document for any researcher interested in studying animals in an exercise context is the Resource Book of the American Physiological Society [12]. It is clear that the study of animals can help elucidate the mechanisms of exercise-related benefits to both human and animal health. Nevertheless, any animal study that has been specifically designed to answer a research question solely on the enhancement of human athletic performance should include a clear explanation as to why such a study is necessary, and why it could not be undertaken on humans. This is important not just from an ethical perspective but is in keeping with the aim of maximising external validity in any study. 


\section{Specific issues relevant to Sports Medicine}

7

1. Retrospective ethics. Data are routinely collected from individuals for various purposes. For example, sport scientists may monitor physiological function of an athlete in order for him or her to gain an edge over their rivals. Data collected exclusively for one purpose cannot be used for another purpose (research) unless consent for the use in research is subsequently given and the research ethically approved. An exception to this would be where the data collected for the primary purpose is anonymised prior to use in a research study (second purpose) which has ethical approval. Retrospective ethical approval cannot be granted for any research study.

2. The use of placebo. The inclusion of a placebo group in a research study may challenge the principle of equipoise. Ideally, participants should be randomly assigned to experimental or placebo groups. In healthy volunteers, where possible, the type of treatment should be blinded, for example, comparator (control) participants could be given a fitness or lifestyle information sheet. After a finite length of time those participants in the comparator group could be offered the experimental condition, or an experiment could be halted if at any point it became clear that the placebo group was fairing more poorly [14]. In more medical research where participants are patients or clients extreme care must be taken to avoid the abuse of placebo. In this type of research, the use of placebo is acceptable when no current proven intervention exists, or when the participant will not be at risk of serious or irreversible harm.

3. Deception. The use of deception in research (e.g. in a pacing strategy study in which time trial distance is deceived) must be merited such that there are no reasonable alternatives for obtaining the data, as long as there is no reasonable expectation to cause pain or severe emotional distress. If deception is to be used, then the participants must be accurately informed of the risks and be debriefed at the conclusion of the study with the option to withdraw their data [15].

4. Good research practice. The UK Medical Research Council has outlined some basic principles of good research practice which may help to ensure that research is conducted ethically. These include planning, conduct, recording data, reporting results, applying the results [13]. Relevant issues not specifically raised in the Declaration of Helsinki include the use, calibration and maintenance of equipment, $\mathrm{COSHH}$, documentation of standard operating procedures, retention of data, publication policy, authorship, correction of errors and retraction of published findings and intellectual property rights.

\section{Update. Athletes as participants in studies on doping agents \\ $\nabla$}

In principle, recreational and elite athletes should not be recruited to participate in research that exposes them to violations of the World Anti-Doping Code. However, the value of research into doping in sport is recognised - as well as the need to use athletes as participants. Investigators who wish to recruit athletes as participants in research involving prohibited performance enhancing substances and methods should consider the following:
I. Consultation with appropriate and relevant authorities (specific to each individual athlete) such as research ethics committees, WADA, international sport federations and national anti-doping organisations - to help protect recreational athletes, elite athletes and sport.

II. An unfair advantage should not be afforded to a recreational or elite athlete participating in the research.

III. "Adequate precautions should be taken so that the results of research are not misused and applied for doping" [16]

IV. Append the WADA letter entitled "Scientific research using elite athletes: WADA point of view" [6] to the participant information sheet to help fully inform participants who are recreational or elite athletes

\section{Update. Ethics and sample size \\ $\nabla$}

Statistical power should be considered by all authors submitting to IJSM. Ideally an a priori estimation of the minimal sample size for adequate statistical power and/or adequate precision of a confidence interval should be reported. Authors and reviewers of IJSM manuscripts should be aware of the following important points:

I. The minimal sample size for adequate statistical power should be considered alongside the burden of the study procedures on individual participants/animals and the predicted importance of the study findings to the knowledge base as well as to the impact on real-world practice [1]. An unethical scenario is where many participants or animals have been substantially burdened by the study procedures, but the study findings have dubious clinical/practical importance. A "small" study might not be unethical, especially if participant burden is low and clinical/practical importance of the study findings are high, even if "statistical significance" has not been realised.

II. For the importance of a study to be judged, it is imperative that the minimal clinically/practically important magnitude of change or difference is rationalised clearly and reported by authors [5]. We encourage authors to report the associated confidence interval(s), at least for the primary study outcomes. Authors who rely solely on statistical significance to judge clinical/practical importance will have their manuscripts rejected.

III. It is inappropriate for a reviewer to criticise a study on the basis of a perceived small sample size without considering the above issues of participant burden and clinical/practical importance.

References

1 Bacchetti P, Wolf LE, Segal MR, McCulloch CE. Ethics and sample size. Am J Epidemiol 2005; 161: 105-110

2 Declaration of Helsinki. World Medical Association.Available at http:// www.wma.net/e/ethics unit/helsinki.htm

3 Harriss DJ, Atkinson G. International Journal of Sports Medicine - ethical standards in sport and exercise science research. Int J Sports Med 2009; 30: 701-702

4 Harriss DJ, Atkinson G. Update - ethical standards in sport and exercise science research. Int J Sports Med 2011; 32: 819-821

5 Hopkins WG, Marshall SW, Batterham AM, Hanin J. Progressive statistics for studies in sports medicine and exercise science. Med Sci Sports Exerc 2009; 41: 3-12

6 Howman D. Scientific research using elite athletes: WADA point of view. J Appl Physiol 2013; 114: 1365 
7 Hughes J, Hunter D, Sheehan M, Wilkinson SD, Wrigley A, Hughes J. European textbook on ethics in research. Luxembourg: Publications Office of the European Union, 2010; 203

8 Institute for Laboratory Animal Research, National Research Council. Guide for the Care and Use of Laboratory Animals. Washington, D.C: National Academy Press, 1996; 140

9 Institute for Laboratory Animal Research, National Research Council. Guide for the Care and Use of Laboratory Animals. Washington, D.C.: National Academy Press, 2010; 248

10 Institute for Laboratory Animal Research, National Research Council. Guide for the care and use of laboratory animals - report in brief. Washington, D.C.: National Academy Press, 2010. Available at http:// dels.nas.edu/resources/static-assets/materials-based-on-reports/ special-products/guide_lab_animals_final.pdf

11 International Sociological Association, Code of Ethics. Madrid: International Sociological Association, 2001. Available at http://www.isasociology.org/about/isa_code_of_ethics.htm
12 Kregel KC. Resource book for the design of animal exercise protocols. Committee to develop an American physiological society resource book for the design of animal exercise protocols. Available via http:// www.the-aps.org/pa/action/exercise/book.pdf. Accessed 24 October 2013

13 Medical Research Council. MRC ethics series: Good research practice 2005. London: Medical Research Council, 2005; 1-16

14 Shephard RJ. Ethics in exercise science research. Sports Med 2002; 32: $169-183$

15 The American Psychological Association. Ethical principles of psychologists and code of conduct. Am Psychol 2002; 57: 1060-1073

16 World Anti Doping Code. World Anti Doping Agency. Available at http:// stage.wada-ama.org/Documents/World_Anti-Doping_Program/ WADP-The-Code/WADA_Anti-Doping_CODE_2009_EN.pdf 